\title{
Decay and detection of a light scalar boson mixing with the Higgs boson
}

\author{
Martin Wolfgang Winkler \\ Nordita, KTH Royal Institute of Technology and Stockholm University, \\ Roslagstullsbacken 23, 10691 Stockholm, Sweden
}

(Received 10 September 2018; published 9 January 2019)

\begin{abstract}
The simplest extension of the standard model consists in adding one singlet scalar field which mixes with the Higgs boson. $\mathcal{O}(\mathrm{GeV})$ masses of the new scalar carry strong motivation from relaxion, dark matter and inflation models. The decay of a GeV scalar is, however, notoriously difficult to address since, at this mass scale, the chiral expansion breaks down and perturbative QCD does not apply. Existing estimates of the GeV scalar decay rate disagree by several orders of magnitude. In this work, we perform a new dispersive analysis in order to strongly reduce these uncertainties and to address discrepancies in earlier results. We will update existing limits on light scalars and future experimental sensitivities which are in some cases strongly affected by the new-found decay rates. The meson form factors provided in this work, can be used to generalize our findings to non-universally coupled light scalars.
\end{abstract}

DOI: 10.1103/PhysRevD.99.015018

\section{INTRODUCTION}

Many prominent extensions of the standard model (SM) feature a gauge singlet scalar $\phi$ with a mass below or at the weak scale. Within the relaxion mechanism [1] the new scalar is introduced to cure the (little) hierarchy problem. In well-motivated dark matter models, a light scalar emerges as the mediator which links the dark and the visible sector [2]. A light scalar appears in supersymmetric theories such as the next-to-minimal supersymmetric standard model [3]. It has been identified with the field driving cosmic inflation [4,5] and it is present in models which address the cosmological constant problem through radiative breaking of classical scale invariance [6].

Through mixing with the Higgs, the light scalar inherits the Higgs couplings to SM matter reduced by a universal suppression factor. While for scalar masses around the electroweak scale, LEP and LHC constraints on extended Higgs sectors apply, rare meson decays offer a particular powerful search channel for scalars below the bottom mass threshold [7]. If the mixing is suppressed, the scalar may, however, travel a macroscopic distance before decay. In this case, searches including missing energy or displaced vertices become relevant. Present and future experimental

\footnotetext{
*martin.winkler@su.se
}

Published by the American Physical Society under the terms of the Creative Commons Attribution 4.0 International license. Further distribution of this work must maintain attribution to the author(s) and the published article's title, journal citation, and DOI. Funded by SCOAP. sensitivities to a light scalar thus crucially depend on its decay rate and decay pattern.

Since the chiral expansion breaks down shortly above the two-pion threshold, while a perturbative QCD calculation becomes reliable for masses of a few $\mathrm{GeV}$, the scalar decay rate in the window $m_{\phi} \simeq 0.5-2 \mathrm{GeV}$ suffers from notorious uncertainties (see e.g., [8]). The problem already manifested itself when a light SM Higgs was still considered viable [9]. In the late 1980s, it was realized that the form factors determining the Higgs (or general scalar) decay rate to meson final states are accessible through dispersion relations [10]. Unfortunately, the two most comprehensive calculations based on this technique by Truong and Willey [11] and Donoghue et al. [12] disagree by orders of magnitude at $m_{\phi} \sim \mathrm{GeV}$. It was argued in [12] that Truong and Willey had obtained the wrong interference pattern between elastic and inelastic contributions to the form factors due to a sign error. In this work, we will reinvestigate the discrepancy and recalculate the decay rate of a light scalar to pions and kaons. Our evaluation profits from progress in the description of pion/kaon phase shift data entering the dispersive integral.

After identifying the favored parameter regions for some of the most promising SM extensions with light scalars, we will update the existing limits and future experimental sensitivities. These were previously based on varying sets of assumptions on the scalar decay. In several cases, we find the sensitivities to be substantially altered by our new-found decay rates. This holds in particular in the context of beam dump experiments which are very sensitive to the scalar decay length through the location of the detector. 


\section{STANDARD MODEL EXTENSIONS WITH LIGHT SCALARS}

A new scalar can connect to the SM at the renormalizable level via the Higgs portal

$$
\mathcal{L} \supset\left(g_{1} \phi+g_{2} \phi^{2}\right)\left(H^{\dagger} H\right) .
$$

Once electroweak symmetry is broken, the couplings $g_{1,2}$ induce mixing between the scalar and the Higgs. We will focus on the case where the scalar mass is considerably below the electroweak scale. In the low energy effective theory, the Higgs can then be integrated out and it arises the coupling of the new scalar to SM fermions

$$
\mathcal{L} \supset-\frac{s_{\theta} m_{f}}{v} \phi \bar{f} f
$$

where $s_{\theta}$ denotes the sine of the Higgs-scalar mixing angle and $v$ the Higgs vacuum expectation value (vev). With regard to experimental searches, the light scalar behaves as a light version of the Higgs boson with universally suppressed couplings. In concrete models, a more complicated coupling pattern may emerge if they feature e.g., more than one Higgs doublet. While we focus on the simplest case given above, many of our results can be applied to more general couplings after simple rescaling. In order to identify the most promising parameter space for the mixing angle, we shall briefly discuss some well-motivated SM extensions with light scalars.

\section{A. Connection to dark matter}

New particles with a weak scale annihilation cross section have been considered among the leading dark matter candidates since-within the thermal production mechanism-their relic density naturally matches the observed dark matter density. The absence of a signal in direct detection experiments, however, suggests even feebler interactions between dark matter and nuclei. An appealing possibility is that dark matter resides within a dark sector of particles which do not directly feel the strong or electroweak forces [2]. In this scenario, a scalar boson could be the mediator which communicates between dark and visible matter. In the simplest realization, dark matter is identified with a gauge singlet Majorana fermion $\chi$ which is stable due to a (discrete) symmetry and couples to the scalar via the Yukawa term $[13,14]$

$$
\mathcal{L} \supset \frac{\kappa}{2} \phi \bar{\chi} \chi
$$

We will assume $m_{\chi}>m_{\phi}$, such that a hierarchy between the annihilation cross section and the dark matter nucleus cross section can naturally be realized: the fermions annihilate into scalars via the (unsuppressed) coupling $\kappa$, while dark matter nucleus interactions are suppressed by the mixing angle $s_{\theta}$. The annihilation cross section times relative velocity $v_{\text {rel }}$ is of the size $\sigma v_{\text {rel }}=\sigma_{1} v_{\text {rel }}^{2}$ with $[13,15]$

$$
\sigma_{1} \simeq \frac{\kappa^{4} m_{\chi}}{24 \pi} \frac{9 m_{\chi}^{4}-8 m_{\chi}^{2} m_{\phi}^{2}+2 m_{\phi}^{4}}{\left(2 m_{\chi}^{2}-m_{\phi}^{2}\right)^{4}} \sqrt{m_{\chi}^{2}-m_{\phi}^{2}},
$$

where we assumed a vanishing trilinear scalar self-coupling for simplicity. ${ }^{1}$ Since the annihilation cross section is p-wave suppressed, strong indirect dark matter detection constraints are avoided. The fermion relic density is approximated as [16]

$$
\Omega_{\chi} h^{2}=2.8 \times 10^{-11} \mathrm{GeV}^{-2} \frac{m_{\chi}^{2}}{\sqrt{g_{*}\left(T_{F}\right)} \sigma_{1} T_{F}^{2}},
$$

where $g_{*}$ denotes the number of relativistic degrees of freedom and $T_{F}$ the freeze-out temperature which we take from [17]. For a given set of masses, the coupling $\kappa$ is fixed by requiring that $\Omega_{\chi} h^{2}$ matches the observed dark matter relic density. We find $\kappa=(0.03-0.05) \times \sqrt{m_{\chi} / \mathrm{GeV}}$ for $m_{\chi}=10 \mathrm{MeV}-10 \mathrm{TeV} .^{2}$

We have implicitly assumed a standard thermal freezeout of the singlet fermion. This is justified if the dark sector was in thermal equilibrium with the SM bath prior to freeze-out. We, therefore, require that the thermalization rate $\Gamma_{\text {therm }}$ of the dark sector exceeds the Hubble rate of expansion $H$ at freeze-out, i.e.,

$$
\Gamma_{\text {therm }}\left(T_{F}\right)>H\left(T_{F}\right)=\sqrt{\frac{4 \pi^{3} g_{*}\left(T_{F}\right)}{45}} \frac{T_{F}^{2}}{\sqrt{8 \pi} M_{P}} .
$$

Since $\Gamma_{\text {therm }}$ scales with $s_{\theta}^{2}$, (6) puts a lower limit on the mixing angle. Notice that thermal decoupling of the dark sector is not a strict exclusion criterion. It would, however, invalidate the simple connection between $m_{\chi}, \kappa$ and $\Omega_{\chi} h$, making the relic density a UV sensitive quantity.

At the same time, large mixing angles are excluded due to direct dark matter detection. The dark matter-nucleon cross section reads ${ }^{3}[14]$

$$
\sigma_{n} \simeq \frac{4 \mu_{\chi}^{2}}{\pi}\left(\frac{s_{\theta} \kappa}{2 v m_{\phi}^{2}} m_{n} f^{n}\right)^{2}
$$

with

$$
f^{n}=f_{u}^{n}+f_{d}^{n}+f_{s}^{n}+\frac{6}{27} f_{G}
$$

\footnotetext{
${ }^{1}$ The general expression for the annihilation cross section for nonvanishing trilinear coupling can be found in [15].

${ }^{2}$ This holds unless for very degenerate cases $m_{\chi}-m_{\phi}<$ $0.01 m_{\chi}$.

${ }^{3}$ The formula is valid for scalar masses substantially larger than the momentum transfer, i.e., $m_{\phi} \gtrsim 100 \mathrm{MeV}$.
} 
Here, $m_{n}$ denotes the nucleon mass and $\mu_{\chi}$ the reduced mass of the dark matter-nucleon system. The scalar coefficients $f_{u, d, s}^{n}$ and $f_{G}$ define the quark and gluon content of the nucleon. While $f_{G}=1-f_{u}^{n}-f_{d}^{n}-f_{s}^{n}$ derives from the QCD trace anomaly, $f_{u, d, s}^{n}$ have been determined in lattice-QCD and in chiral perturbation theory. We employ the value $f^{n}=0.30$ from [18] which is consistent with other recent evaluations $[19,20]$. The dark matter direct detection constraints can now be mapped into the scalar mass-mixing plane. Besides the constraints of XENON1T [21], we also include those of CRESST-III [22] and DarkSide-50 [23] which dominate at $m_{\chi} \lesssim 5 \mathrm{GeV}$.

Since the most conservative (weakest) bounds are obtained if $\chi$ is just slightly heavier than $\phi$, we fix $m_{\chi}=1.1 m_{\phi}$. In this case, the thermalization rate is dominated by the inverse decay of the scalar [24] and we have to apply (6) with $\Gamma_{\text {therm }} \simeq \Gamma_{\phi}$. As shown in Fig. 8, the parameter space, where the correct relic density can be achieved via standard freeze-out and the direct detection constraints are satisfied, spans several orders of magnitude in $s_{\theta}$. Further experimental constraints on this window will be discussed in Sec. IV.

\section{B. Relaxion}

The relaxion mechanism constitutes a dynamical solution to the (little) hierarchy problem of the standard model [1]. It provides another motivation for the existence of a light scalar boson. While the phenomenology of Higgsrelaxion mixing has been comprehensively studied [25,26], we wish to include the additional possibility of a low inflationary Hubble scale $H_{I}$.

The evolution of the relaxion $\phi$ reduces the initially large Higgs boson mass $M \gg v$ to the observed mass $m_{h}=\mathcal{O}(v)$. This is achieved via the potential ${ }^{4}$

$V=\left(M^{2}-g M \phi\right) h^{2}-g M^{3} \phi-\Lambda^{2} h^{2} \cos \left(\frac{\phi}{f}\right)+\lambda h^{4}$,

where $g$ is a dimensionless coupling and $h$ denotes the neutral component of the Higgs doublet. Since the relaxion settles in a $C P$ breaking minimum, it is not identified with the QCD axion in the basic model. Instead, the periodic potential may stem from the instantons of a new strongly coupled gauge group [1]. ${ }^{5}$ The scale $\Lambda$ must not exceed the electroweak scale since, otherwise, the Higgs vev is driven up to $\Lambda$. This constraint also ensures that a constant term in front of the cosine, which is generated by closing the Higgs

\footnotetext{
${ }^{4}$ We neglect an $\mathcal{O}(1)$ coefficient in front of the $g M^{3}$ term which does not play a role for the following discussion.

${ }^{5}$ For concreteness, we assumed that the new strongly coupled sector does not break electroweak symmetry such that odd powers of $h$ are absent in front of the cosine. The phenomenology is, however, hardly sensitive to this assumption (see [26]).
}

loop, is sufficiently suppressed and does not trap the relaxion before electroweak symmetry breaking $[25,26]$.

The relaxion slowly rolls down its potential and, at $\phi \sim$ $M / g$ triggers electroweak symmetry breaking. As soon as the Higgs field is displaced, the cosine term induces wiggles on the relaxion potential which ultimately stop its motion. The required dissipation mechanism is provided by the Hubble friction of inflation. If $H_{I}$ exceeds a critical value $H_{I, c} \sim \sqrt{g M^{3} / f}$, the relaxion immediately stops in one of its first minima. Otherwise, it continues rolling and later settles in one of the steeper minima, further down the potential. The difference between both cases manifests in the phase factor

$$
\sin \left(\frac{v_{\phi}}{f}\right) \sim \operatorname{Min}\left(1, \frac{H_{I}^{2} f}{g M^{3}}\right),
$$

where we introduced the relaxion vev $v_{\phi}$. The sine is of order unity if $H_{I}>H_{I, c}$, while it can be substantially suppressed for a low inflationary scale. The Higgs vev emerges as

$$
v^{2} \simeq \frac{g M^{3} f}{\Lambda^{2} \sin \left(\frac{v_{\phi}}{f}\right)}
$$

Validity of the effective theory (9) without further light degrees of freedom requires $f \gg v \gtrsim \Lambda$. This implies that the relaxion is lighter than the Higgs and the mixing effect on $m_{h}$ is negligible. The relaxion mass ${ }^{6}$ and the Higgsrelaxion mixing angle can be approximated as [26]

$$
\begin{aligned}
m_{\phi}^{2} & \simeq \frac{\Lambda^{2} v^{2}}{2 f^{2}}\left[\cos \left(\frac{v_{\phi}}{f}\right)-\frac{2 \Lambda^{2}}{m_{h}^{2}} \sin ^{2}\left(\frac{v_{\phi}}{f}\right)\right], \\
s_{\theta} & \simeq \frac{\Lambda^{2} v}{f m_{h}^{2}} \sin \left(\frac{v_{\phi}}{f}\right) .
\end{aligned}
$$

The relaxion couples to SM matter via its Higgs admixture and via pseudoscalar couplings which are generically present but model-dependent. Requiring that the mixinginduced couplings dominate leads to the constraint $\sin \left(v_{\phi} / f\right) \gtrsim 1 /\left(16 \pi^{2}\right) .^{7}$ The resulting theory exclusion on the parameter space (requiring also $f>v$ ) is depicted in Fig. 8. Compared to $[25,26]$, we obtain a larger relaxion window since suppression of $s_{\theta}$ by small $H_{I}$ has not been considered in these references.

\footnotetext{
${ }^{6}$ More precisely, we are referring to the mass of the relaxionlike scalar mass eigenstate.

${ }^{7}$ For $\sin \left(v_{\phi} / f\right) \gtrsim 1 /\left(16 \pi^{2}\right)$, the $C P$ violating scalar relaxion couplings can still dominate since pseudoscalar couplings may suffer additional loop suppression [26]. We note that viable relaxion models with smaller $\sin \left(v_{\phi} / f\right)$ may exist. The constraint, however, singles out the parameter region in which the relaxion can be described as a minimal singlet scalar mixing with the Higgs.
} 


\section{SCALAR DECAY RATES}

It is straightforward to evaluate the scalar decay rates into leptonic final states. One finds

$$
\Gamma(\phi \rightarrow \bar{\ell} \ell) \equiv \Gamma_{\bar{\ell} \ell}=\frac{s_{\theta}^{2} G_{F} m_{\phi}}{4 \sqrt{2} \pi} m_{\ell}^{2} \beta_{\ell}^{3},
$$

with $\ell=e, \mu, \tau$. Here, $G_{F}$ denotes the Fermi constant and $\beta_{\ell}=\sqrt{1-4 m_{\ell}^{2} / m_{\phi}^{2}}$ the velocity of the final state leptons. Hadronic decay rates require a more careful treatment due to the strong final state interactions. This holds in particular, if the scalar mass resides in the vicinity of the $f_{0}(980)$ resonance.

\section{A. Status of hadronic decay rates}

Figure 1 shows that different evaluations of the scalar decay rate to pions disagree by several order of magnitude at $m_{\phi} \sim \mathrm{GeV}$. The result of Voloshin was obtained at leading order in chiral perturbation theory (ChPT) [27]. In the "Higgs Hunter's guide" the perturbative spectator model is extrapolated into the nonperturbative regime. Quark masses were adjusted such as to (approximately) reproduce Voloshin's decay rate at low mass [9]. Both evaluations are frequently used to describe $\mathrm{GeV}$ scalar decays although they do not apply to this mass range due to its proximity to the chiral symmetry breaking scale. Raby and West [10] introduced the use of dispersion relations to access the $\mathrm{GeV}$ regime and predicted a huge enhancement of the scalar decay rate to pions close to the $f_{0}(980)$ resonance. However, they treated $f_{0}(980)$ as an elastic $\pi \pi$-resonance which leads to an overestimation of the rate. A full two-channel analysis including $K K$ and $\pi \pi$ was finally performed by Truong and Willey [11] and Donoghue et al. [12]. Their results are incompatible with one another, which was related to a sign error in Truong and Willey's $T$-matrix parametrization in [12]. Monin et al. [28] recently performed a modified one-channel analysis in order to provide an analytic expression for $\Gamma_{\pi \pi}$ in terms of the $\pi \pi$-scattering phase. Since free parameters were chosen

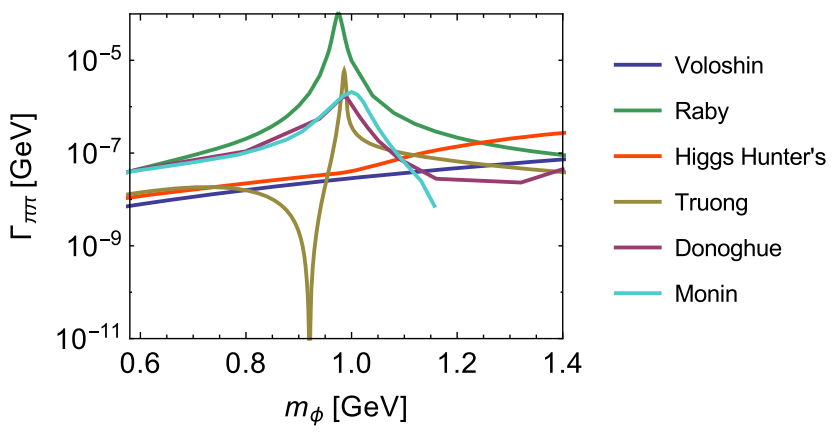

FIG. 1. Evaluations of the light scalar decay rate to pions by Voloshin [27], Raby and West [10], the Higgs Hunter's Guide [9], Truong and Willey [11], Donoghue et al. [12] and Monin et al. [28]. In this figure $s_{\theta}$ has been set to unity. with the purpose of reproducing the rate of Donoghue et al., it was not meant as a test of previous results. A calculation of the hadronic decay rates in an independent two-channel dispersive analysis is still missing. It will be performed in the next sections, before matching the result to the perturbative spectator model at higher mass.

\section{B. Chiral perturbation theory}

We first consider scalar masses below the charm threshold. The Lagrangian describing the interaction of the scalar with light quarks $(u, d, s)$ and gluons reads

$$
\begin{aligned}
\mathcal{L} & \supset s_{\theta} \frac{\phi}{v}\left(\frac{3 \alpha_{s}}{12 \pi} G_{\mu \nu}^{a} G^{a \mu \nu}-m_{u} \bar{u} u-m_{d} \bar{d} d-m_{s} \bar{s} s\right) \\
& =-s_{\theta} \frac{\phi}{v}\left(\frac{2}{9} \Theta_{\mu}^{\mu}+\frac{7}{9}\left(m_{u} \bar{u} u+m_{d} \bar{d} d+m_{s} \bar{s} s\right)\right),
\end{aligned}
$$

where the effective coupling to gluons origins from heavy quark $(c, b, t)$ loops. In the second step, we used the trace identity

$$
\Theta_{\mu}^{\mu}=-\frac{9 \alpha_{s}}{8 \pi} G_{\mu \nu}^{a} G^{a \mu \nu}+m_{u} \bar{u} u+m_{d} \bar{d} d+m_{s} \bar{s} s,
$$

of the energy-momentum tensor which results from the conformal anomaly $[29,30]$. The decay rates of the scalar into pion and kaon pairs read

$$
\begin{gathered}
\Gamma_{\pi \pi}=\frac{3 s_{\theta}^{2} G_{F}}{16 \sqrt{2} \pi m_{\phi}} \beta_{\pi}\left|\frac{7}{9} \Gamma_{\pi}+\frac{7}{9} \Delta_{\pi}+\frac{2}{9} \Theta_{\pi}\right|^{2}, \\
\Gamma_{K K}=\frac{s_{\theta}^{2} G_{F}}{4 \sqrt{2} \pi m_{\phi}} \beta_{K}\left|\frac{7}{9} \Gamma_{K}+\frac{7}{9} \Delta_{K}+\frac{2}{9} \Theta_{K}\right|^{2},
\end{gathered}
$$

where we introduced the form factors

$$
\begin{aligned}
\Gamma_{\pi} & =\left\langle\pi \pi\left|m_{u} \bar{u} u+m_{d} \bar{d} d\right| 0\right\rangle, \\
\Delta_{\pi} & =\left\langle\pi \pi\left|m_{s} \bar{s} s\right| 0\right\rangle, \\
\Theta_{\pi} & =\left\langle\pi \pi\left|\Theta_{\mu}^{\mu}\right| 0\right\rangle,
\end{aligned}
$$

for pions and analogous for kaons. The pion form factors have been determined to lowest order in ChPT in [27]. A ChPT calculation of the kaon form factors may seem pointless since the scalar decay to kaons only opens in the regime, where chiral symmetry is strongly broken. However, the low-momentum kaon form factors will later define the matching conditions for the dispersive analysis. Therefore, we briefly outline the computation using the (strangeness-conserving part of the) 3-flavor chiral Lagrangian which reads ${ }^{8}$

\footnotetext{
${ }^{8} \mathrm{An}$ analogous determination of the kaon form factors can be found in [12]. For a review on the application of ChPT techniques to Higgs physics, see [9,31].
} 


$$
\mathcal{L}=\frac{1}{4} f_{\pi} \operatorname{Tr} \partial_{\mu} \Sigma \partial^{\mu} \Sigma^{\dagger}+\frac{1}{2} f_{\pi}^{2}\left(\operatorname{Tr} \mu M \Sigma^{\dagger}+\text { H.c. }\right),
$$

with

$$
\Sigma=\exp \left\{\frac{\sqrt{2} i}{f_{\pi}}\left(\begin{array}{ccc}
\frac{\pi^{0}}{\sqrt{2}}+\frac{\eta}{\sqrt{6}} & \pi^{+} & K^{+} \\
\pi^{-} & -\frac{\pi^{0}}{\sqrt{2}}+\frac{\eta}{\sqrt{6}} & K^{0} \\
K^{-} & \bar{K}_{0} & -\frac{2 \eta}{\sqrt{6}}
\end{array}\right)\right\}
$$

and $M=\operatorname{diag}\left(m_{u}, m_{d}, m_{s}\right)$. Here $f_{\pi}$ denotes the pion decay constant. The mass parameters in the chiral Lagrangian are related to the physical meson masses as

$$
\begin{aligned}
m_{\pi}^{2} & =\mu\left(m_{u}+m_{d}\right), \\
m_{K^{0}}^{2} & =\mu\left(m_{d}+m_{s}\right), \\
m_{K^{ \pm}}^{2} & =\mu\left(m_{u}+m_{s}\right) .
\end{aligned}
$$

One can now use the Feynman-Hellmann theorem $[32,33] m_{q} \bar{q} q=-m_{q} \partial \mathcal{L} / \partial m_{q}$ and the trace of the energy-momentum tensor

$$
\Theta_{\mu}^{\mu}=\frac{f_{\pi}}{2} \operatorname{Tr} \partial_{\mu} \Sigma \partial^{\mu} \Sigma^{\dagger}-g_{\mu}^{\mu} \mathcal{L},
$$

to evaluate the form factors at lowest order in the chiral expansion (denoted by the superscript 0 ). One finds

$$
\begin{aligned}
& \Gamma_{\pi}^{0}=m_{\pi}^{2}, \quad \Gamma_{K}^{0}=\frac{1}{2} m_{\pi}^{2}, \\
& \Delta_{\pi}^{0}=0, \quad \Delta_{K}^{0}=m_{K}^{2}-\frac{1}{2} m_{\pi}^{2}, \\
& \Theta_{\pi}^{0}=s+2 m_{\pi}^{2}, \quad \Theta_{K}^{0}=s+2 m_{K}^{2},
\end{aligned}
$$

where we set $m_{u}=m_{d}$. The form factors have to be evaluated at $\sqrt{s}=m_{\phi}$. Higher orders are suppressed by powers of the chiral symmetry breaking scale $\Lambda_{\chi} \sim 1 \mathrm{GeV}$. The lowest order does, hence, not provide a realistic estimate of the form factors for $m_{\phi} \gtrsim 0.5 \mathrm{GeV}$.

\section{Dispersive analysis}

Fortunately, form factors at higher mass are accessible through dispersion relations. These employ analyticity and unitarity conditions without relying on any details of the microscopic interaction theory. For $\sqrt{s} \lesssim 1.3 \mathrm{GeV}$ a twochannel approximation in terms of $\pi \pi$ and $K K$ can be applied. This is because scalar decays are controlled by the $f_{0}(980)$ resonance at $\sqrt{s} \sim \mathrm{GeV}$ which mainly couples to these states [34]. At even lower mass, where also $f_{0}(500)$ contributes, $\pi \pi$ is the only relevant decay channel due to kinematics.

We define $F=\left(F_{\pi}, \frac{2}{\sqrt{3}} F_{K}\right)(F=\Gamma, \Delta, \Theta)$, where the Clebsch-Gordan coefficient occurring in the isoscalar projection of the $\pi \pi$ state has been absorbed into the definition of $F$ [12]. Below the kaon threshold, the phase of the pion form factors coincides with the isoscalar s-wave $\pi \pi$ phase shift according to Watson's theorem [35]. Its generalization to two channels is expressed in form of the unitary relation

$$
\operatorname{Im} F_{i}=T_{i j}^{*} \beta_{j} F_{j} \theta\left(s-4 m_{j}^{2}\right)
$$

with $\beta_{1,2}=\beta_{\pi, K}$. The (isoscalar s-wave projection of the) $T$-matrix for $\pi \pi, K K \rightarrow \pi \pi, K K$ scattering is parametrized in terms of two phases $\delta, \psi$ and an inelasticity parameter $g$

$$
T=\left(\begin{array}{cc}
\frac{\eta e^{2 i \delta}-1}{2 i \beta_{\pi}} & g e^{i \psi} \\
g e^{i \psi} & \frac{\eta e^{2 i(\psi-\delta)}-1}{2 i \beta_{K}}
\end{array}\right),
$$

where

$$
\begin{aligned}
\eta & =\sqrt{1-4 \beta_{\pi} \beta_{K} g^{2} \theta\left(s-4 m_{K}^{2}\right)}, \\
\beta_{i} & =\sqrt{1-\frac{4 m_{i}^{2}}{s}} .
\end{aligned}
$$

The parameters of the $T$ matrix are efficiently determined by invoking $\pi \pi, K K$ scattering data and theoretical constraints in form of the Roy-Steiner equations. We extract the phases and inelasticity parameter from the analysis of Hoferichter et al. [36] which incorporates earlier results [37,38]. Above $\sqrt{s_{0}}=1.3 \mathrm{GeV}$, the correct asymptotic behavior of the $T$-matrix is ensured by guiding $\delta, \psi$ smoothly to $2 \pi$. We follow [39] and assume that the difference between the phases and their asymptotic values in this regime decreases as

$$
\frac{2}{1+\left(\frac{\sqrt{s}}{\sqrt{s_{0}}}\right)^{m}}
$$

where $m$ is set to 3 . We have verified that form factors at $\sqrt{s}<\sqrt{s_{0}}$ are rather insensitive to the particular function by which the phases approach their asymptotic values. Above $\sqrt{s_{0}}$ the form factors obtained from the two-channel analysis are anyway less trustable since further channels such as $4 \pi, \eta \eta$ become relevant.

Form factors satisfying the unitary relation (23) can be expressed as $[40,41]$

$$
F=\left(\begin{array}{ll}
\Omega_{11} & \Omega_{12} \\
\Omega_{21} & \Omega_{22}
\end{array}\right)\left(\begin{array}{l}
P_{1} \\
P_{2}
\end{array}\right),
$$

where $P_{1,2}$ are polynomials and $\left(\Omega_{11}, \Omega_{21}\right),\left(\Omega_{12}, \Omega_{22}\right)$ are the two linear independent solution-vectors fulfilling the dispersion relation 


$$
\operatorname{Re} F(s)=\frac{1}{\pi}-f_{4 m_{\pi}^{2}}^{\infty} \mathrm{d} s^{\prime} \frac{\operatorname{Im} F\left(s^{\prime}\right)}{s^{\prime}-s} .
$$

The $\Omega_{i j}$ (which are found as described in [39]) are conveniently normalized such that $\Omega_{11}(0)=\Omega_{22}(0)=1$, $\Omega_{12}(0)=\Omega_{21}(0)=0$.

The form factors $\Gamma_{i}, \Delta_{i}$ are expected to vanish at high energy due to the composite nature of mesons. Since $\Omega_{i j} \propto$ $s^{-1}$ for large $s$, the polynomial prefactors in (27) need to be constants. Their values can be determined by matching (27) to the lowest order result in chiral perturbation theory (22) at $s=0$. In the case of the energy-momentum form factors, Lorentz-invariance and four-momentum conservation require the structure [42]

$$
\Theta_{i}=\frac{3}{2} s \Theta_{S, i}+\left(2 m_{i}^{2}-\frac{s}{2}\right) \Theta_{T, i} \quad(i=\pi, K),
$$

where $\Theta_{S, i}$ and $\Theta_{T, i}$ refer to the scalar and tensor parts of $\Theta_{i}$. In order to match the chiral result at $s=0$, one needs to require that $\Theta_{S, i}, \Theta_{T, i}$ (rather than $\Theta_{i}$ ) vanish asymptotically (see also [12]). We thus obtain

$$
\begin{aligned}
\Gamma_{\pi} & =m_{\pi}^{2}\left(\Omega_{11}+\frac{1}{\sqrt{3}} \Omega_{12}\right), \\
\Delta_{\pi} & =\frac{2}{\sqrt{3}}\left(m_{K}^{2}-\frac{m_{\pi}^{2}}{2}\right) \Omega_{12}, \\
\Theta_{\pi} & =\left(2 m_{\pi}^{2}+p s\right) \Omega_{11}+\frac{2}{\sqrt{3}}\left(2 m_{K}^{2}+q s\right) \Omega_{12}, \\
\Gamma_{K} & =\frac{m_{\pi}^{2}}{2}\left(\sqrt{3} \Omega_{21}+\Omega_{22}\right), \\
\Delta_{K} & =\left(m_{K}^{2}-\frac{m_{\pi}^{2}}{2}\right) \Omega_{22}, \\
\Theta_{K} & =\frac{\sqrt{3}}{2}\left(2 m_{\pi}^{2}+p s\right) \Omega_{21}+\left(2 m_{K}^{2}+q s\right) \Omega_{22},
\end{aligned}
$$

where we introduced

$$
\begin{aligned}
& p=1-2 m_{\pi}^{2} \Omega_{11}^{\prime}(0)-\frac{4 m_{K}^{2}}{\sqrt{3}} \Omega_{12}^{\prime}(0), \\
& q=1-\sqrt{3} m_{\pi}^{2} \Omega_{21}^{\prime}(0)-2 m_{K}^{2} \Omega_{22}^{\prime}(0) .
\end{aligned}
$$

Numerically, we find $p=0.73$ and $q=0.52$. The most relevant SU(3) breaking effect (concerning the impact on decay rates) applies to the value of $q$ which can get changed by up to 0.2 [12]. In Fig. 2 we depict the pion and kaon form factors resulting from our dispersive analysis. The corresponding scalar decay rate to pions is shown in Fig. 3. We also provide an estimate of the uncertainties related to the phase extrapolation and to the matching conditions. The error band was obtained by varying $m$ in (26) between 2 and 4 , and $q$ in the range $0.32-0.72$. Note that uncertainties
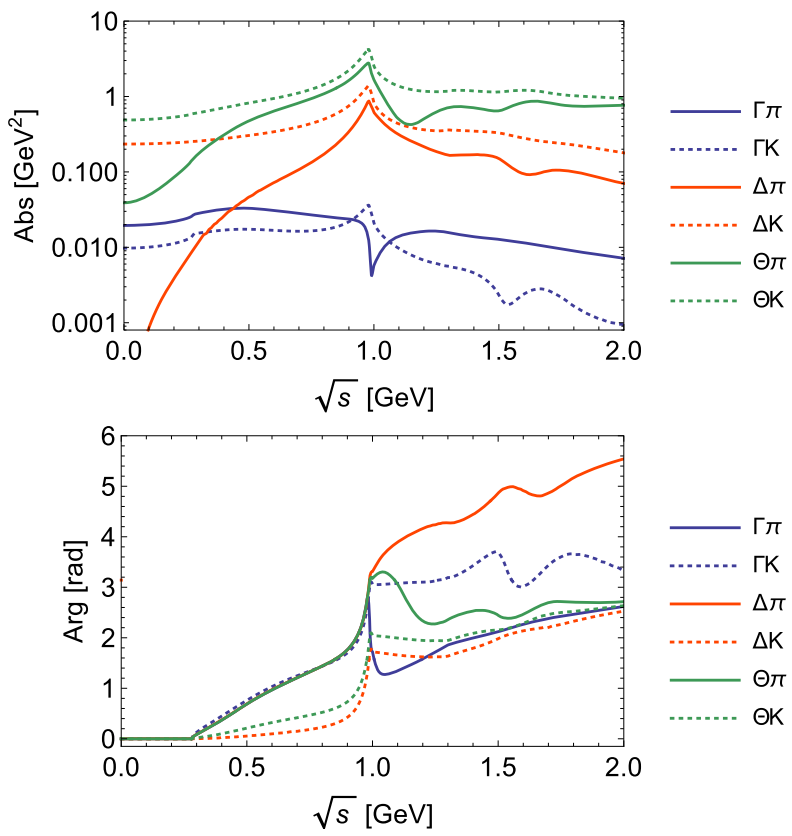

FIG. 2. Modulus (upper panel) and phase (lower panel) of the pion and kaon form factors.

due to the opening of further hadronic decay channels beyond $\pi \pi$ and $K K$ are not included.

As can be seen in the same figure, our result on the decay rate agrees reasonably well with that of Donoghue et al. [12]. Differences reside within a factor of $\sim 3$ and follow from our updated phase shift input [36]. The decay rates found by us are, however, incompatible with those in [11]. The reason for the discrepancy is indeed a sign error in Truong and Willey's parametrization of the $T$-matrix. Their choice leads to a negative sign of $T_{12}$ at low energy which is inconsistent with ChPT [12]. In Fig. 3 we also depict the decay rate after flipping the sign of their parameter $\lambda$. It can be seen that this correction puts Truong and Willey's rate into qualitative agreement with our result.

\section{Perturbative spectator model}

We now turn to the hadronic decays at higher energy, where the perturbative spectator model can be applied. The decay rates to quarks are given as ${ }^{9}$

$$
\Gamma_{\bar{\ell} \ell}: \Gamma_{\bar{s} s}: \Gamma_{\bar{c} c}=m_{\ell}^{2} \beta_{\mu}^{3}: 3 m_{s}^{2} \beta_{K}^{3}: 3 m_{c}^{2} \beta_{D}^{3}
$$

and analogous for the $\bar{b} b$-channel. The kinematic threshold is set by the lightest meson containing an $s$ or $c$ quark respectively [9]. In addition, we need to consider the loopinduced decay rate into gluon pairs [43]

\footnotetext{
${ }^{9}$ We set $m_{s}=95 \mathrm{MeV}, m_{c}=1.3 \mathrm{GeV}$ [34] and neglect the tiny decay rate into $u, d$ quarks.
} 

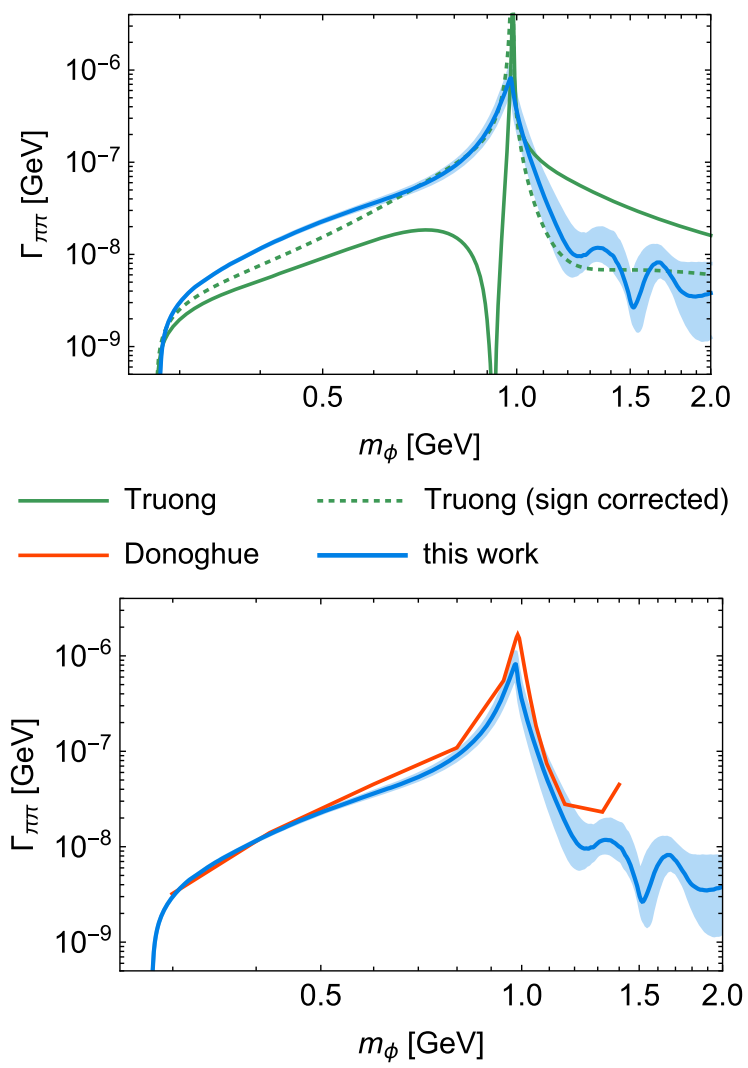

FIG. 3. Light scalar decay rate into pions from this work, from Truong \& Willey [11] and from Donoghue et al. [12]. We also show Truong and Willey's decay rate after correcting a sign error in their $T$-matrix parametrization (see text).

$$
\Gamma_{g g}=\frac{s_{\theta}^{2} \alpha_{s}^{2} m_{\phi}^{3}}{32 \pi^{3} v^{2}}\left|\sum_{\text {quarks }} \frac{x_{i}+\left(x_{i}-1\right) f\left(x_{i}\right)}{x_{i}^{2}}\right|^{2},
$$

with $x_{i}=m_{\phi}^{2} /\left(4 m_{i}^{2}\right)$ and

$$
f(x)= \begin{cases}\arcsin ^{2} \sqrt{x}, & x \leq 1 \\ -\frac{1}{4}\left(\log \frac{1+\sqrt{1-1 / x}}{1-\sqrt{1-1 / x}}-\mathrm{i} \pi\right)^{2}, & x>1 .\end{cases}
$$

We take $\alpha_{s}\left(m_{\phi}\right)$ from [44]. Following [45] we assume that the perturbative spectator model is valid at $m_{\phi}>2 \mathrm{GeV}$. The dispersive analysis holds for $m_{\phi} \lesssim 1.3 \mathrm{GeV}$, where $\pi \pi$ and $K K$ dominate the hadronic decay rate. In the regime $m_{\phi}=1.3-2 \mathrm{GeV}$, significant corrections are expected. We will use the dispersive results up to $2 \mathrm{GeV}$, but include an additional contribution

$$
\Gamma_{4 \pi, \eta \eta, \rho \rho, \ldots}=C s_{\theta}^{2} m_{\phi}^{3} \beta_{2 \pi}
$$

to account for the increasing number of hadronic channels opening above the $4 \pi$ threshold. The mass scaling is leaned upon the gluon channel. Setting $C=5.1 \times 10^{-9} \mathrm{GeV}^{-2}$, the hadronic decay rate transits smoothly into the rate of the spectator model at $m_{\phi}=2 \mathrm{GeV}$.

In reality, peaks may occur in different hadronic channels due to further unflavored scalar resonances including $f_{0}(1370), f_{0}(1500), f_{0}(1710), f_{0}(2020), f_{0}(2100)$, $f_{0}(2200)$ and $f_{0}(2300)$ [46]. The strong increase of the decay rate around $\mathrm{GeV}$, however, appears since $f_{0}(980)$ is narrow and located just below the kaon threshold to which it strongly couples [11]. Since a comparable situation does not seem to arise for the listed heavier $f_{0}$-resonances, less pronounced enhancements are expected at higher mass. Therefore, the hadronic decay rates we obtain at $m_{\phi}=$ 1.3-2 GeV may at least provide a valid order-of-magnitude estimate. On the other hand, presently unconfirmed

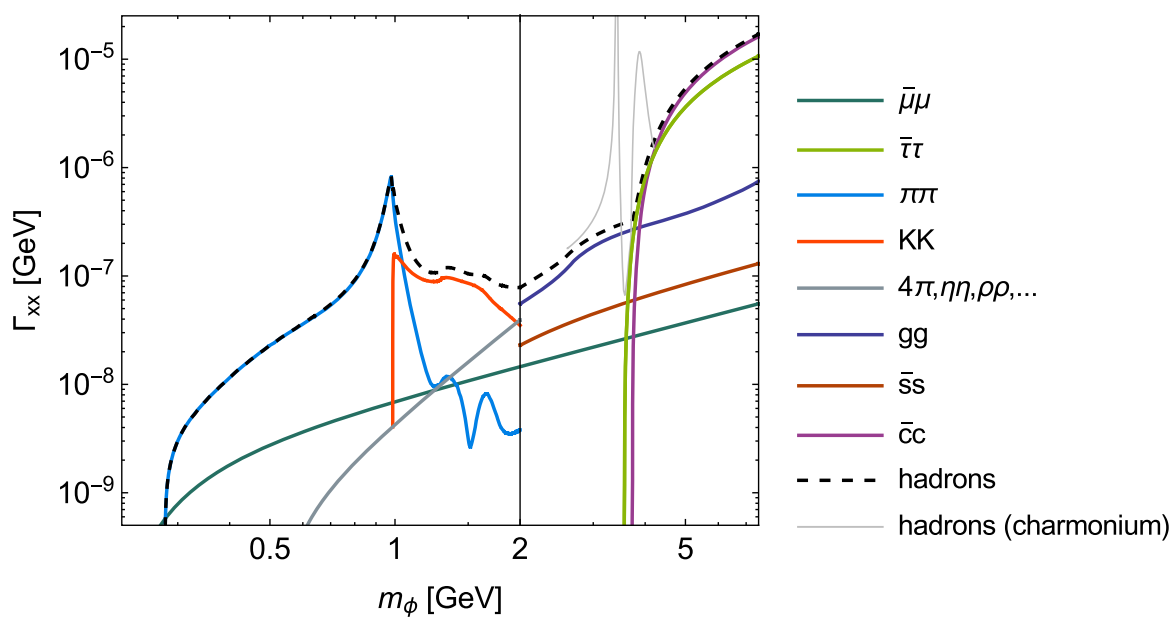

FIG. 4. Hadronic and leptonic decay rates of a light scalar mixing with the Higgs. These were obtained from our dispersive analysis $\left(m_{\phi}<2 \mathrm{GeV}\right)$ and from the perturbative spectator model $\left(m_{\phi}>2 \mathrm{GeV}\right)$. The possible impact of the charmonium resonances on the hadronic decay rate is illustrated by the gray line. All decay rates scale with $s_{\theta}^{2}$ which was set to unity in this plot. 
resonances including potential glueball states could still have significant impact [47].

Around the two-charm threshold, the scalar mixes with the $C P$ even quarkonia $\chi_{c 0}(n P)$. In Appendix $\mathrm{A}$, we assess the effect on the scalar decay rates due to $\chi_{c 0}(1 P)$ and $\chi_{c 0}(2 P)$ within a nonrelativistic potential approach [48]. Since nonperturbative corrections to the simple quantum mechanical picture are unknown ${ }^{10}$ and due to the sparseness of experimental data on the heavier charmonium resonances, this should only be seen as a very qualitative estimate. For masses $m_{\phi} \sim 10 \mathrm{GeV}$, which are not in the main focus of this work, the bottomonium resonances cause analogous mixing effects [48].

In Fig. 4, we depict the leptonic and hadronic decay rates of the light scalar below the $\bar{b} b$-threshold. These were obtained from the dispersive results matched to the spectator model as described above. The possible distortion of the hadronic decay rate due to the charmonium resonances (see Appendix A) is also indicated for illustration. Due to the mentioned uncertainties, we will mask the regions $m_{\phi}=3.3-3.5 \mathrm{GeV}$ and $m_{\phi}=3.75-4.0$ around the charmonium peaks in the experimental analysis.

\section{EXPERIMENTAL CONSTRAINTS AND FUTURE SENSITIVITIES}

Experimental limits on light scalars as well as future sensitivities have been summarized various times, recently in $[24,26,50-53]$. These crucially depend on the decay properties of the scalar. In many instances, constraints with different assumptions on the hadronic decay rate have been combined. We will, therefore, reevaluate the existing limits on light scalars consistently using our new set of decay rates. Sensitivities of some important future searches will also be discussed. Our focus is on the mass window $m_{\phi} \simeq$ $0.01-9.8 \mathrm{GeV}$ which is accessible to accelerator probes (and below the threshold of bottomonium resonances).

\section{A. Rare decays}

Light scalars can mediate rare meson decays. The most relevant processes include radiative $\Upsilon$-decays as well as flavor changing $B$ and $K$ meson decays (see Fig. 5). The calculation of the corresponding branching ratios is summarized in Appendix B.

$B A B A R$ has performed various searches for radiative $\Upsilon$ decays mediated by a light scalar. The most important channel is $\gamma \rightarrow \gamma+$ jets triggered by a hadronically decaying $\phi$ [54]. ${ }^{11}$

Below the $B$ threshold, searches for semileptonic $B$ decays become relevant. $\mathrm{LHCb}$ measured the branching

\footnotetext{
${ }^{10}$ The decay width of $\chi_{c 0}(1 P)$ is e.g., strongly underestimated in the potential models which might suggest the importance of instanton effects [49].

${ }^{11}$ For $m_{\phi} \gtrsim 4 \mathrm{GeV}$ a very similar sensitivity to light scalars is achieved in the channel $\Upsilon \rightarrow \gamma+\bar{\tau} \tau$ [55].
}
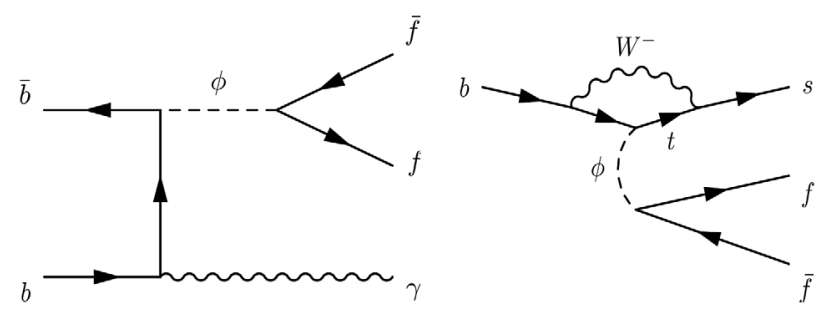

FIG. 5. Radiative $\Upsilon$ decays and flavor changing $B$ decays mediated by a light scalar.

ratio $B^{+} \rightarrow K^{+}+\bar{\mu} \mu$ in several bins of dilepton invariant mass [56]. The corresponding upper limit on the $\phi$-induced branching ratio in each bin is determined as in [14]. It must be taken into account that LHCb triggered on prompt decays in this search. Following [14], we estimate that events with a (boosted) scalar decay length $d<d_{\max } \simeq 5 \mathrm{~mm}$ are reconstructed. This translates to an efficiency factor

$$
\eta=\int_{0}^{\infty} \mathrm{d} p_{\phi} f\left(p_{\phi}\right)\left(1-e^{-m_{\phi} \Gamma_{\phi} d_{\max } / p_{\phi}}\right),
$$

where $f\left(p_{\phi}\right)$ denotes the momentum distribution of $\phi$ which is obtained with PYTHIA [57]. ${ }^{12}$ LHCb has subsequently performed dedicated searches for light scalars with macroscopic decay lengths. In [58,59] constraints on $\mathrm{Br}_{B^{0} \rightarrow K^{* 0} \phi} \times$ $\mathrm{Br}_{\phi \rightarrow \bar{\mu} \mu}$ and $\mathrm{Br}_{B^{+} \rightarrow K^{+} \phi} \times \mathrm{Br}_{\phi \rightarrow \bar{\mu} \mu}$ have been set as a function of the intermediate scalar mass and lifetime. We digitized the provided images and derived the corresponding constraints on $s_{\theta} \cdot{ }^{13}$ As can be seen in Fig. 6, the inclusion of displaced decays has significantly increased the LHCb sensitivity to light scalars in most of the mass range. A search for longlived particles in $B$ decays was also performed by $B A B A R$ which looked for the inclusive process $B \rightarrow X_{s} \phi$ with $\phi$ further decaying into leptons or hadrons [60]. The pion channel is most relevant since it excludes a small parameter region not covered by the previously mentioned $\mathrm{LHCb}$ searches.

Scalar masses of up to a few hundred $\mathrm{MeV}$ can be probed by rare kaon decays. We include the upper limit $\mathrm{Br}_{K_{L} \rightarrow \pi^{0}+\bar{\mu} \mu}<3.8 \times 10^{-10}$ stemming from the $\mathrm{KTeV}$ experiment [61] in our analysis. Again, $\phi$-mediated processes only contribute to the rate if $\phi$ decays sufficiently promptly. Since $\mathrm{KTeV}$ is a fixed-target experiment, event reconstruction mostly depends on the transverse vertex location. Following [62] we assume that events with a (boosted) transverse scalar decay length below $4 \mathrm{~mm}$ pass the trigger. The corresponding efficiency factor is calculated using (36) with $p_{\phi}$ replaced by the transverse

\footnotetext{
${ }^{12}$ We generated a large sample of $B$ mesons with PYTHIA and decayed each $B$ further to $\phi$ using the appropriate kinematics.

${ }^{13}$ The case of a light scalar mixing with the Higgs has been covered explicitly in the two references. We, nevertheless, rederive the constraints on $s_{\theta}$ since a different set of scalar decay rates has been employed in $[58,59]$.
} 


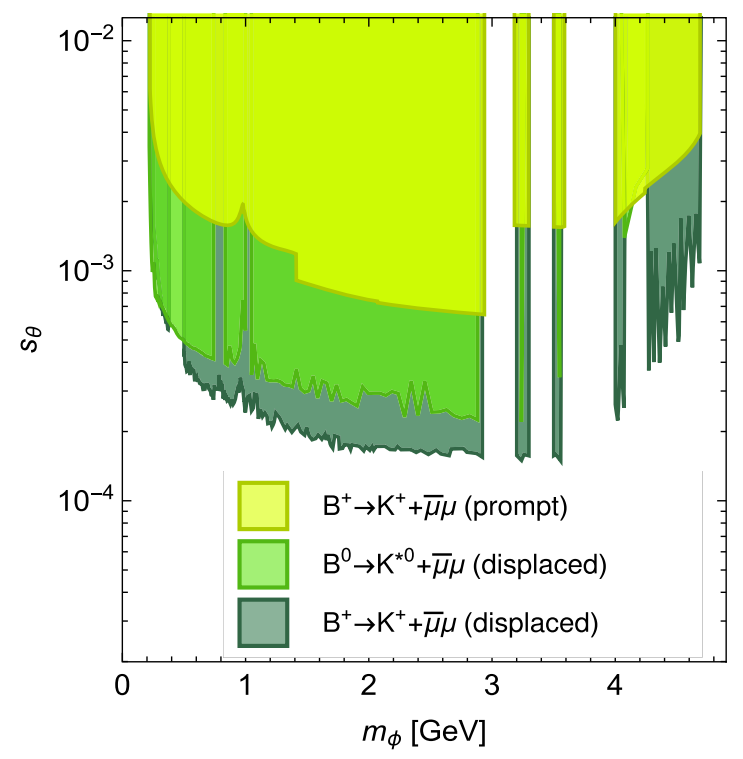

FIG. 6. LHCb constraints on light scalars derived from rare $B$ decays. Masses around the charmonium resonances have been masked by the collaboration or by us (see Sec. III D).

momentum. The distribution of transverse momenta is again determined with PYTHIA.

Below the muon threshold, the scalar typically escapes detection due to its long lifetime. It still leaves a trace in the form of missing energy. The search for $K^{+} \rightarrow \pi^{+}+\bar{\nu} \nu$ by E949 is used to set limits on $\mathrm{Br}_{K^{+} \rightarrow \pi^{+} \phi}$ as a function of the scalar mass and lifetime [63]. In this case, visible decays of $\phi$ are vetoed, and the sensitivity increases with the lifetime of the scalar. We determine the corresponding exclusion in the $m_{\phi}-s_{\theta}$ plane. The standard model contribution to $K^{+} \rightarrow \pi^{+}+\bar{\nu} \nu$ is neglected for this purpose.

Figure 8 shows that rare decays set the strongest constraints on light scalars over wide regions of the parameter space. Mixing angles down to $s_{\theta}=10^{-3}-10^{-4}$ are excluded for $m_{\phi}<m_{B}-m_{K}$ unless $m_{\phi}$ resides in the vicinity of the charmonium resonances. The limits substantially degrade once scalar production in $B$ decays becomes kinematically inaccessible.

\section{B. Collider searches}

At LEP, searches for Higgs bosons and Higgs-like scalars have been performed through the process $\bar{e} e \rightarrow Z^{*} \phi$. In the considered mass window, the strongest constraints are set by $L 3$ [64]. Strictly speaking, these apply to scalars which share the exact decay properties of a SM Higgs boson (at the considered mass). Since the mixing angle $s_{\theta}$ suppresses the couplings of $\phi$ compared to the Higgs one may worry that the longer decay length invalidates the bounds. This is not the case: for the range of $s_{\theta} \gtrsim$ 0.1 covered by the search, a light SM Higgs and a light scalar would both decay mostly invisibly (meaning outside the detector) below the muon threshold and visibly above.
Even in the $\mathrm{GeV}$ range (and beyond), the $L 3$ analysis can be considered robust since it merely relies on the dominance of hadronic decay modes, while the particular enhancement of the pionic decay rate does not play a role. Above the $B$ meson mass, LEP still sets the strongest constraints on light scalars (see Fig. 8).

Turning to the LHC, light scalars are constrained by the search for spin-0 resonances in the dimuon channel. CMS and LHCb provided constraints on $\sigma_{p p \rightarrow \phi} \times \mathrm{Br}_{\phi \rightarrow \bar{\mu} \mu}$ at $\sqrt{s}=7 \mathrm{TeV}$ and $8 \mathrm{TeV}$ respectively $[65,66]$ (see also [67]). In the covered mass range $m_{\phi}=5.5-15 \mathrm{GeV}$, scalar production by $B$-meson decay is kinematically forbidden which makes gluon fusion the relevant process. We calculated the corresponding cross section with the tool SUSHI 1.6.1 [68] in order to translate the limits into exclusions on $s_{\theta}$ for our considered mass range (see Fig. 8). Additional LHC constraints on light scalars arise from the nonobservation of exotic Higgs decays. These shall not be considered in this work since they rely on the modeldependent Higgs-scalar coupling and, furthermore, only lead to subdominant exclusions in the considered mass range [26]. For proposed detector concepts (MATHUSLA, CODEX-b, FASER) which would increase the LHC sensitivity to light scalars, we refer to $[24,52,53]$.

\section{Beam dump experiments}

Beam dump experiments with detectors located $\mathcal{O}(100 \mathrm{~m})$ away from the interaction point provide a sensitive laboratory to search for long-lived particles. Light scalars are most efficiently generated by $B$ and $K$ meson decays. For a proton beam impinging on a thick target which absorbs hadrons efficiently, the number of produced scalars can be estimated as

$$
N_{\phi} \simeq N_{\text {p.o.t. }}\left(n_{B} \mathrm{Br}_{B \rightarrow X_{s} \phi}+\left\langle\gamma_{K}^{-1}\right\rangle \ell_{H} n_{K} \Gamma_{K \rightarrow \pi \phi}\right)
$$

with $N_{\text {p.o.t. }}$ denoting the number of protons on target. The multiplicities $n_{B}, n_{K}$ stand for the number of $B, K$ mesons created per incoming proton, $\left\langle\gamma_{K}^{-1}\right\rangle$ for the mean inverse kaon Lorentz factor. In the case of kaons only $K^{ \pm}$and $K_{L}$ should be considered since $K_{S}$ has a suppressed decay rate to scalars (see Appendix B 3). Different from $B$ mesons, most kaons are absorbed in the target since their decay length exceeds the hadronic absorption length $\ell_{H}{ }^{14}$ The above approximation neglects kaon regeneration by secondary interactions. Furthermore, it assumes that the number of kaons escaping the target is negligible (as is valid for a target with a thickness of several $\ell_{H}$ ).

The probability $\mathcal{P}_{\phi}$ that a scalar with three-momentum $\mathbf{p}_{\phi}$ leaves a signal in the detector reads

\footnotetext{
${ }^{14}$ While the decay length differs substantially between $K^{ \pm}$and $K_{L}, \Gamma_{K}^{-1} \gg \ell_{H}$ holds for both species.
} 


$$
\mathcal{P}_{\phi}=\int_{d_{1}}^{d_{2}} \mathrm{~d} z \frac{\eta_{\mathrm{geom}} \eta_{\mathrm{rec}} m_{\phi} \Gamma_{\phi}}{p_{\phi}} e^{-m_{\phi} \Gamma_{\phi} z / p_{\phi}}
$$

The decay vertex $z$ of the scalar needs to be located within the distance $d_{1}-d_{2}$ from the target to be detected. The geometric efficiency $\eta_{\text {geom }}$ accounts for the probability that the decay products of $\phi$ pass through the detector. It depends on the angular coverage of the detector and varies with the scalar's momentum and the location $z$ of the decay vertex. The factor $\eta_{\text {rec }}$ is the reconstruction efficiency for final states of a certain type. In order to determine the total number of events, we need to integrate the product $N_{\phi} \mathcal{P}_{\phi}$ over the momentum distribution of $\phi$. The latter is again determined with PYTHIA by creating large samples of $B$ and $K$ mesons which are then decayed further to scalars. Kaon events are properly weighted to account for the fact that highly boosted kaons are more likely to be absorbed due to their longer decay length. The geometric efficiency is determined from the momentum spectrum of $\phi$ by decaying the scalars and selecting events with all final states passing through the detector.

We consider the CHARM beam dump (which operated in the 1980s), the upcoming run of NA62 in dump mode and the planned SHiP experiment. All three detectors have been/ will be located at the CERN SPS and employ a $400 \mathrm{GeV}$ proton beam. ${ }^{15}$ The meson multiplicities are estimated as $n_{B} \simeq 3.2 \times 10^{-7}$ [70] and $n_{K} \simeq 0.9$ [71]. ${ }^{16}$ The target materials copper (CHARM, NA62) and molybdenum (SHiP) share a hadronic absorption length $\ell_{H} \simeq 15.3 \mathrm{~cm}$ [72]. Locations and coverage of the detectors are described in $[70,73,74]$. CHARM is sensitive to leptonic final states with efficiency 0.5 [73]. SHiP and NA62 should be sensitive to all sorts of final states with $\eta_{\text {rec }}=0.4$ $\left(\eta_{\text {rec }}=0.7\right)$ below (above) the two-muon threshold for SHiP [70] and $\eta_{\text {rec }} \simeq 1$ for NA62 [75]. We summarize the luminosities, locations of the decay volumes and mean geometric efficiencies $\bar{\eta}_{\text {geom }}$ (for detection of $B$ - and $K$-induced scalars) in Table I. ${ }^{17}$ SHiP will be a factor $\mathcal{O}\left(10^{4}\right)$ more sensitive compared to its predecessors due to the larger beam intensity and the better detector coverage.

CHARM did not observe any signal events which translates to an upper limit of 3 expected events (at 95\% confidence level). The corresponding exclusion on light scalars reaches down to $s_{\theta} \sim 10^{-4}$ (see Fig. 8). We note that the CHARM constraint obtained by

\footnotetext{
${ }^{15}$ A search for long-lived scalars could potentially also be performed at the Fermilab SeaQuest Experiment after minor modifications of the setup [69].

${ }^{16}$ We extracted $n_{K^{ \pm}}=0.62$ from [71] and estimated $n_{K_{L}} \simeq$ 0.28 by taking the $K_{L} / K^{ \pm}$ratio from PYTHIA.

${ }^{17}$ The mean geometric efficiency $\bar{\eta}_{\text {geom }}$ was derived by averaging $\eta_{\text {geom }}$ over the momentum distribution and the location of the decay vertex within $d_{1}-d_{2}$. The stated ranges are obtained by varying the scalar mass between $0.01 \mathrm{GeV}$ and $m_{B}-m_{K}$.
}

TABLE I. Comparison between the CHARM, NA62 and SHiP beam dump experiments.

\begin{tabular}{lccc}
\hline \hline & $\mathrm{N}_{\text {p.o.t }}$ & $d_{1}-d_{2}[\mathrm{~m}]$ & $\bar{\eta}_{\text {geom }}$ \\
\hline CHARM & $2.4 \times 10^{18}$ & $480-515$ & $0.001-0.002(K)$ \\
& & & $0.002-0.01(B)$ \\
NA62 & $10^{18}$ & $95-160$ & $0.002-0.005(K)$ \\
& & & $0.002-0.02(B)$ \\
SHiP & $2 \times 10^{20}$ & $69-120$ & $0.05-0.08(K)$ \\
& & & $0.2-0.5(B)$ \\
\hline \hline
\end{tabular}

us is substantially weaker than in previous evaluations $[8,24,26,50-53,62]$. We believe that in these references, kaon absorption in the thick copper target-which drastically reduces $N_{\phi}$ from kaon decays- has been neglected.

Sensitivity projections for NA62 and SHiP in Fig. 8 again correspond to 3 events. They should be considered as optimistic since a negligible background level was assumed. While the number of produced scalars in NA62 is similar as in CHARM, NA62 is sensitive to higher masses since it can reconstruct pion final states. SHiP will cover a huge parameter region not previously accessible to any experiment. For SHiP and NA62, we again find deviations from the semi-official sensitivity estimates [75,76] (see Fig. 7).

In this case, the discrepancy can be traced back to the assumptions on the scalar decay rates. While we relied on a dispersive analysis in the non-perturbative QCD regime (see Sec. III), the perturbative spectator model with

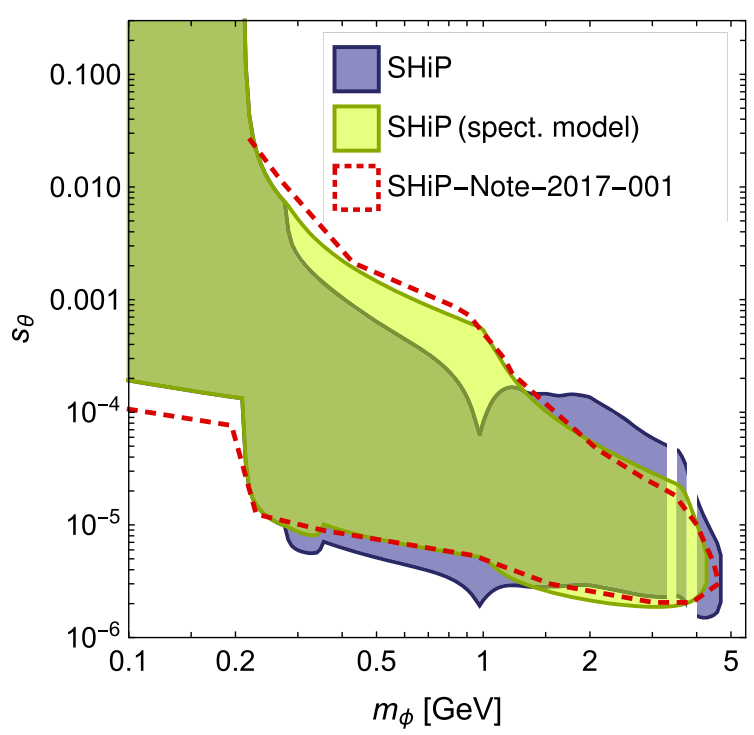

FIG. 7. SHiP sensitivity to light scalars found in this work compared to [76]. The blue shaded region is obtained for the scalar decay rates derived in Sec. III and represents our preferred estimate. The yellow region is obtained if we treat the scalar decay in the perturbative spectator model. 

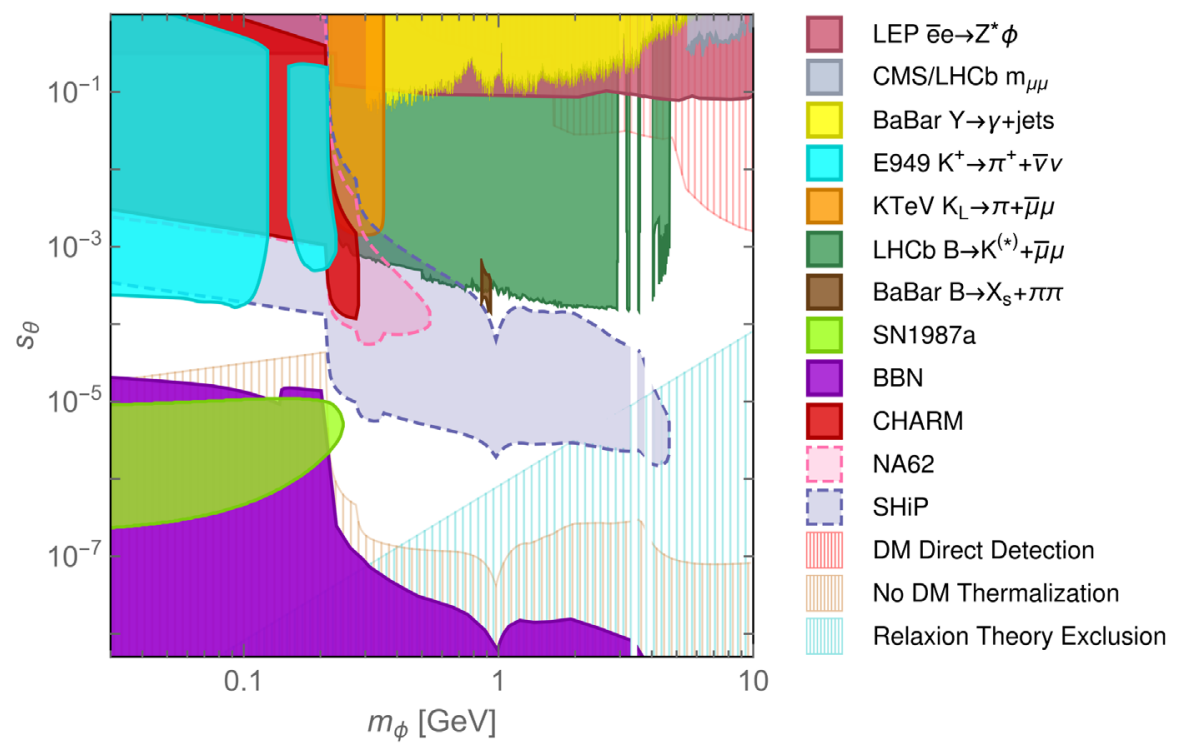

FIG. 8. Constraints on light scalars mixing with the Higgs. The filled regions with solid boundaries correspond to model-independent constraints. Sensitivity projections are indicated by the dashed boundary. The hatched regions refer to model-dependent exclusions which apply to the relaxion model (cyan) and the dark matter model (red, ocher) discussed in Sec. II. Masses around the charmonium resonances have been masked in some probes.

adjusted quark masses [9] has been employed in [75,76]. In Fig. 7 it can be seen that our sensitivity estimate approximately reproduces the SHiP projection from [76] if we also switch to the same spectator model. The same observation is made in the case of NA62. We emphasize, however, that our dispersive analysis provides a much more realistic description of the scalar decay properties in the $\mathrm{GeV}$ range compared to the spectator model.

We finally comment that the sensitivity of NA62 to light scalars could be significantly improved: the present estimate refers to the experiment running in dump mode. This means that the beryllium target is lifted and the collimator is closed such that it acts as dump for the proton beam. The disadvantage of this layout is that most produced kaons are absorbed in the thick collimator before they can decay. It appears preferential to leave the (thin) beryllium target in the beam line and keep the collimator closed. The latter would then still filter hadronic backgrounds. But since it is located $20 \mathrm{~m}$ downstream the target, a significant fraction of the kaons created in the target could decay before reaching the collimator. This would increase the number of light scalars from kaon decay by a factor 10-100 compared to dump mode.

\section{Cosmology and astrophysics}

Light scalars can also be constrained by requiring that they do not spoil the cosmological evolution. In the hot early universe, the light scalars are copiously produced in the thermal bath. Due to their small coupling to SM matter, their freeze-out abundance is significant. If their decay happens after the onset of primordial nucleosynthesis
(BBN), the hadronic energy injection would have spoiled the light element abundances. The resulting upper limit on the scalar lifetime ranges from $1 / 100 \mathrm{~s}-1 \mathrm{~s}$ in the considered mass range [77]. ${ }^{18}$ It was converted to a constraint on $s_{\theta}$ by using the decay rate from Fig. 4.

Finally, astrophysical processes can be affected by light scalars. Most importantly, scalar emission could carry away significant amounts of energy in supernova explosions [78,79]. This would lead to a shortening of the neutrino pulse which is constrained by observations of SN1987a. We determine the corresponding exclusions on light scalars following the treatment described in [24,51].

While accelerator searches exclude large mixing angles, cosmology constrains $s_{\theta}$ from below (see Fig. 8). For $m_{\phi} \lesssim 5 \mathrm{GeV}$, a window of $s_{\theta} \sim 10^{-3}-10^{-5}$ and $s_{\theta} \sim$ $10^{-4}-10^{-8}$ remains viable below and above the two-muon threshold respectively. In models, where the light scalar is identified with the relaxion (Sec. II B) or the mediator connecting to dark matter (Sec. II A), additional constraints apply which close parts of this window. Nevertheless, there remains an exciting discovery potential for the next generation of experiments.

\section{CONCLUSION}

We have reinvestigated the decay properties of a light scalar boson mixing with the Higgs. A special focus was

\footnotetext{
${ }^{18}$ The constraint mildly depends on the (model-dependent) Higgs-scalar coupling and was shown for three different choices in [77]. To be conservative we used the weakest of the three constraints at each mass.
} 
placed on the mass range $m_{\phi} \simeq 0.5-2 \mathrm{GeV}$ in which hadronic decay modes are affected by strong final state interactions. We performed an updated dispersive analysis and derived the decay rates of the scalar to pions and kaons. These were confronted with two earlier evaluations [11,12] which are inconsistent with one another. Our calculation confirms the result of Donoghue et al. [12] to within $\mathcal{O}(1)$ precision. The remaining difference can be explained by our updated input of pion-kaon phase shift data. Truong and Willey's decay rate [11] differs substantially from ours due to a sign error in their $T$-matrix parametrization, first pointed out in [12]. We flipped the sign in their calculation and showed that this, indeed, brings their result into qualitative agreement with our findings. By matching the dispersive calculation to the perturbative spectator model at higher mass, we then obtained — with the mentioned caveats-a realistic estimate of scalar decay rates over the full mass range (Fig. 4). We also provided the hadronic form factors which allow to generalize our result to nonuniversally coupled light scalars (Fig. 2).

Finally, we rederived the accelerator-, cosmological and theoretical constraints on light scalars in the $\mathrm{MeV}-\mathrm{GeV}$ mass window (Fig. 8). We covered the model-independent case as well some of the most prominent explicit models with light scalars. Sensitivity projections for future key searches were also provided. The strongest deviations compared to previous evaluations occur for beam dump experiments. In the case of CHARM, previous exclusions were too restrictive since they had neglected kaon absorption in the target. In addition, our new-found decay rates strongly impact the sensitivity window of beam dumps by affecting the decay length of light scalars.

\section{ACKNOWLEDGMENTS}

I would like to thank Kai Schmidt-Hoberg, Felix Kahlhöfer, Katherine Freese, Luca Visinelli and Sebastian Baum for helpful discussions.

\section{APPENDIX A: MIXING WITH THE CHARMONIUM RESONANCES}

We estimate the impact of the charmonium resonances on the scalar decay rates in a nonrelativistic potential approach [48]. For this purpose, we consider the $\chi_{c 0}(1 P)$ state and the $\chi_{c 0}(2 P)$ candidate. Masses and widths are set to the central values listed in [46]. Notice, however, that the uncertainty on $\Gamma_{\chi_{c 0}(2 P)}$ reaches almost an order of magnitude. Two higher $\chi_{c 0}$ resonances are listed as candidates for exotic structure [46] and can likely not be described within simple potential models.

The off-diagonal mass matrix element between $\phi$ and $\chi_{c 0}(n P)$ is estimated as [48],

$$
m_{\phi \chi_{c 0}}^{2}=s_{\theta} \sqrt{\frac{27 \sqrt{2}}{\pi} G_{F} m_{\chi_{c 0}}}\left|R_{\chi_{c 0}}^{\prime}(0)\right| .
$$

The derivative of the radial quarkonium wave function at the origin can be extracted from the measured decay rate $\Gamma_{\chi_{c 0}(1 P) \rightarrow \gamma \gamma}=2.0 \mathrm{keV}[80]$. We find $\left|R_{\chi_{c 0}(1 P)}^{\prime}(0)\right|^{2} \simeq$ $0.06 \mathrm{GeV}^{5}$ and assume the same value for the heavier state. The angles characterizing the mixing of the scalar with the two charmonium states are approximated as [48]

$$
\vartheta_{1,2} \simeq \frac{m_{\phi \chi_{c 0}}^{2}}{m_{\phi}^{2}-m_{\chi_{c 0}}^{2}+i m_{\chi_{c 0}} \Gamma_{\chi_{c 0}}} .
$$

The mixing-induced contributions to the scalar decay amplitude derive from the meson decay amplitudes multiplied by $\vartheta_{1,2}$. These are added to the perturbative amplitudes from which the charm part is subtracted in order to avoid double counting. The interference between perturbative and mixing-induces terms depends on the assumptions regarding the charmonium branching ratios (see [48]). We set $\operatorname{Br}\left(\chi_{c 0}(1 P) \rightarrow g g\right)=1$ for the lighter resonance which is located below the $D D$-threshold. For the heavier state, we assume $\operatorname{Br}\left(\chi_{c 0}(2 P) \rightarrow D D\right)=0.95$, $\operatorname{Br}\left(\chi_{c 0}(2 P) \rightarrow g g\right)=0.05$ (such that the decay rate to gluons is similar for both resonances). The resulting hadronic scalar decay rate for this estimate is shown in Fig. 4.

\section{APPENDIX B: SCALAR IN RARE DECAYS}

\section{Radiative $\Upsilon$ decays}

A light scalar can emerge in the radiative decay $\gamma \rightarrow \gamma \phi$ and induce a meson or lepton pair [81]. It is convenient to express the corresponding branching ratio in the form

$$
\frac{\mathrm{Br}_{\Upsilon \rightarrow \gamma \phi}}{\mathrm{Br}_{\Upsilon \rightarrow \bar{e} e}}=\frac{s_{\theta}^{2} G_{F} m_{b}^{2}}{\sqrt{2} \pi \alpha} \mathcal{F}\left(1-\frac{m_{\phi}^{2}}{m_{\Upsilon}^{2}}\right),
$$

where $\alpha$ is the Sommerfeld constant and $\mathcal{F}$ a correction function taken from [82]. It accounts for higher order QCD processes $[83,84]$ as well as bound state effects appearing close to the kinematic endpoint $[85,86]$.

\section{Rare $B$ decays}

The scalar appears in an effective flavor violating coupling $\phi$-s-b. By integrating out the $W$-t-loop one obtains [87]

$$
\begin{aligned}
\mathcal{L}_{\phi s b} & =g_{\phi s b} \phi \bar{s}_{L} b_{R}+\text { H.c. }, \\
g_{\phi s b} & =\frac{s_{\theta} m_{b}}{v} \frac{3 \sqrt{2} G_{F} m_{t}^{2} V_{t s}^{*} V_{t b}}{16 \pi^{2}},
\end{aligned}
$$


where $V_{t s}$ and $V_{t b}$ denote the Cabibbo-KobayashiMaskawa matrix elements. The above Lagrangian triggers the decay $B \rightarrow K^{(*)} \phi$ for which the rate reads

$$
\Gamma_{B \rightarrow K^{(*)} \phi}=\left|g_{\phi s b}\right|^{2}\left|\left\langle K^{(*)}\left|\bar{s}_{L} b_{R}\right| B\right\rangle\right|^{2} \frac{\lambda_{B, K^{(*)} \phi}^{1 / 2}}{16 \pi m_{B}},
$$

where we introduced

$$
\lambda_{x, y z}=\frac{m_{x}^{2}-\left(m_{y}-m_{z}\right)^{2}}{m_{x}^{2}} \frac{m_{x}^{2}-\left(m_{y}+m_{z}\right)^{2}}{m_{x}^{2}}
$$

The matrix elements can be approximated as $[88,89]$

$$
\begin{aligned}
\left|\left\langle K^{*}\left|\bar{s}_{L} b_{R}\right| B\right\rangle\right|^{2} & =\frac{1}{4} \frac{m_{B}^{4} \lambda_{B, K^{(*)} \phi}}{\left(m_{b}+m_{s}\right)^{2}} A_{K^{*}}^{2}, \\
\left|\left\langle K\left|\bar{s}_{L} b_{R}\right| B\right\rangle\right|^{2} & =\frac{1}{4} \frac{\left(m_{B}^{2}-m_{K}^{2}\right)^{2}}{\left(m_{b}-m_{s}\right)^{2}} f_{K}^{2}
\end{aligned}
$$

with

$$
\begin{aligned}
A_{K^{*}} & =\frac{1.36}{1-q^{2} / 27.9 \mathrm{GeV}^{2}}-\frac{0.99}{1-q^{2} / 36.8 \mathrm{GeV}^{2}}, \\
f_{K} & =\frac{0.33}{1-q^{2} / 37.5 \mathrm{GeV}^{2}} .
\end{aligned}
$$

The transferred momentum is set to $q^{2}=m_{\phi}^{2}$. In the case of $K^{*}$ we already took the sum over polarizations.

For cases where the nature of the strange particle(s) in the final state is not of relevance, one can define the inclusive decay rate $B \rightarrow X_{s} \phi$. The spectator model predicts [7]

$$
\Gamma_{B \rightarrow X_{s} \phi}=\left|g_{\phi s b}\right|^{2} \frac{\left(m_{B}^{2}-m_{\phi}^{2}\right)^{2}}{32 \pi m_{B}^{3}} .
$$

This estimate is not valid close to the kinematic endpoint, where the spectator model breaks down. In this regime, the inclusive rate should, however, converge towards $\Gamma_{B \rightarrow K \phi}$ since this is the only available final state. In order to obtain a smooth function with the correct asymptotic behavior, we use (B7) for $m_{\phi}<4.7 \mathrm{GeV}$ and set $\Gamma_{B \rightarrow X_{s} \phi}=$ $\Gamma_{B \rightarrow K \phi}$ above.

\section{Rare $K$ decays}

The scalar can also induce rare decays of lighter mesons, for instance $K \rightarrow \pi \phi$. The corresponding decay rate is again dominated by the $W$-t-loop. One finds ${ }^{19}$ [91]

$$
\Gamma_{K^{ \pm} \rightarrow \pi^{ \pm} \phi} \simeq\left|g_{\phi d s}\right|^{2}\left|\left\langle\pi\left|\bar{d}_{L} s_{R}\right| K\right\rangle\right|^{2} \frac{\lambda_{K, \pi \phi}^{1 / 2}}{16 \pi m_{K}},
$$

and $\Gamma_{K_{L} \rightarrow \pi^{0} \phi} \simeq \Gamma_{K^{ \pm} \rightarrow \pi^{ \pm} \phi}$. The effective coupling $g_{\phi d s}$ is obtained from (B2) by the replacement $(b, s) \rightarrow(s, d)$. The matrix element reads [92]

$$
\left|\left\langle\pi\left|\bar{d}_{L} s_{R}\right| K\right\rangle\right| \simeq \frac{1}{2} \frac{\left(m_{K}^{2}-m_{\pi}^{2}\right)}{m_{s}-m_{d}} .
$$

Since the corresponding rate for the $K_{S}$ decays is proportional to the small $C P$ violating phase in the Cabibbo-Kobayashi-Maskawa matrix, it suffers a stronger suppression [90].

\footnotetext{
${ }^{19}$ We neglect subleading contributions due to scalar bremsstrahlung and the charm loop which would amount to a correction $\lesssim 10 \%$ [90].
}

[1] P. W. Graham, D. E. Kaplan, and S. Rajendran, Phys. Rev. Lett. 115, 221801 (2015).

[2] M. Pospelov, A. Ritz, and M. B. Voloshin, Phys. Lett. B 662, 53 (2008).

[3] P. Fayet, Nucl. Phys. B90, 104 (1975).

[4] M. Shaposhnikov and I. Tkachev, Phys. Lett. B 639, 414 (2006).

[5] F. Bezrukov and D. Gorbunov, J. High Energy Phys. 05 (2010) 010.

[6] R. Foot and A. Kobakhidze, Int. J. Mod. Phys. A 30, 1550126 (2015).

[7] R. S. Willey and H. L. Yu, Phys. Rev. D 26, 3086 (1982).

[8] J. D. Clarke, R. Foot, and R. R. Volkas, J. High Energy Phys. 02 (2014) 123.
[9] J. F. Gunion, H. E. Haber, G. L. Kane, and S. Dawson, Front. Phys. 80, 1 (2000).

[10] S. Raby and G. B. West, Phys. Rev. D 38, 3488 (1988).

[11] T. N. Truong and R.S. Willey, Phys. Rev. D 40, 3635 (1989).

[12] J. F. Donoghue, J. Gasser, and H. Leutwyler, Nucl. Phys. B343, 341 (1990).

[13] R. Kappl, M. Ratz, and M. W. Winkler, Phys. Lett. B 695, 169 (2011).

[14] K. Schmidt-Hoberg, F. Staub, and M. W. Winkler, Phys. Lett. B 727, 506 (2013).

[15] M. W. Winkler, Ph.D. thesis, Technical University of Munich, 2012.

[16] M. Drees, M. Kakizaki, and S. Kulkarni, Phys. Rev. D 80, 043505 (2009). 
[17] G. Steigman, B. Dasgupta, and J. F. Beacom, Phys. Rev. D 86, 023506 (2012).

[18] G. Belanger, F. Boudjema, A. Pukhov, and A. Semenov, Comput. Phys. Commun. 185, 960 (2014).

[19] M. Hoferichter, J. Ruiz de Elvira, B. Kubis, and U.-G. Meiner, Phys. Rev. Lett. 115, 092301 (2015).

[20] M. Hoferichter, P. Klos, J. Menndez, and A. Schwenk, Phys. Rev. Lett. 119, 181803 (2017).

[21] E. Aprile et al. (XENON Collaboration), Phys. Rev. Lett. 121, 111302 (2018).

[22] F. Petricca et al. (CRESST Collaboration), arXiv:1711. 07692.

[23] P. Agnes et al. (DarkSide Collaboration), Phys. Rev. Lett. 121, 081307 (2018).

[24] J. A. Evans, S. Gori, and J. Shelton, J. High Energy Phys. 02 (2018) 100.

[25] K. Choi and S. H. Im, J. High Energy Phys. 12 (2016) 093.

[26] T. Flacke, C. Frugiuele, E. Fuchs, R. S. Gupta, and G. Perez, J. High Energy Phys. 06 (2017) 050.

[27] M. B. Voloshin, Yad. Fiz. 44, 738 (1986) [Sov. J. Nucl. Phys. 44, 478 (1986)].

[28] A. Monin, A. Boyarsky, and O. Ruchayskiy, arXiv: 1806.07759 [Phys. Rev. D (to be published)].

[29] R. J. Crewther, Phys. Rev. Lett. 28, 1421 (1972).

[30] M. S. Chanowitz and J. R. Ellis, Phys. Lett. 40B, 397 (1972).

[31] S. Dawson and H. E. Haber, Int. J. Mod. Phys. A 07, 107 (1992).

[32] H. Hellmann, Einführung in die Quantenchemie (Deuticke, Leipzig, 1937) [Introduction to Quantum Chemistry (Deuticke, Leipzig, 1937)].

[33] R. P. Feynman, Phys. Rev. 56, 340 (1939).

[34] C. Patrignani et al. (Particle Data Group), Chin. Phys. C 40, 100001 (2016).

[35] K. M. Watson, Phys. Rev. 95, 228 (1954).

[36] M. Hoferichter, C. Ditsche, B. Kubis, and U. G. Meissner, J. High Energy Phys. 06 (2012) 063.

[37] I. Caprini, G. Colangelo, and H. Leutwyler, Eur. Phys. J. C 72, 1860 (2012).

[38] P. Buettiker, S. Descotes-Genon, and B. Moussallam, Eur. Phys. J. C 33, 409 (2004).

[39] B. Moussallam, Eur. Phys. J. C 14, 111 (2000).

[40] N. I. Muskhelishvili and J. R. M. Radok, Singular integral equations: Boundary problems of function theory and their application to mathematical physics (Noordhoff, Groningen, 1953).

[41] R. Omnes, Nuovo Cimento 8, 316 (1958).

[42] J. F. Donoghue and H. Leutwyler, Z. Phys. C 52, 343 (1991).

[43] M. Spira, A. Djouadi, D. Graudenz, and P. M. Zerwas, Nucl. Phys. B453, 17 (1995).

[44] S. Bethke, Prog. Part. Nucl. Phys. 58, 351 (2007).

[45] B. Grinstein, L. J. Hall, and L. Randall, Phys. Lett. B 211, 363 (1988).

[46] M. Tanabashi et al. (Particle Data Group), Phys. Rev. D 98, 030001 (2018).

[47] S. Narison, Phys. Lett. B 228, 513 (1989).

[48] M. Drees and K.-i. Hikasa, Phys. Rev. D 41, 1547 (1990).

[49] M. B. Voloshin, Prog. Part. Nucl. Phys. 61, 455 (2008).

[50] S. Alekhin et al., Rep. Prog. Phys. 79, 124201 (2016).
[51] G. Krnjaic, Phys. Rev. D 94, 073009 (2016).

[52] J. L. Feng, I. Galon, F. Kling, and S. Trojanowski, Phys. Rev. D 97, 055034 (2018).

[53] J. A. Evans, Phys. Rev. D 97, 055046 (2018).

[54] J. P. Lees et al. (BABAR Collaboration), Phys. Rev. Lett. 107, 221803 (2011).

[55] J. P. Lees et al. (BABAR Collaboration), Phys. Rev. D 88, 071102 (2013).

[56] R. Aaij et al. (LHCb Collaboration), J. High Energy Phys. 02 (2013) 105.

[57] T. Sjstrand, S. Ask, J. R. Christiansen, R. Corke, N. Desai, P. Ilten, S. Mrenna, S. Prestel, C. O. Rasmussen, and P. Z. Skands, Comput. Phys. Commun. 191, 159 (2015).

[58] R. Aaij et al. (LHCb Collaboration), Phys. Rev. Lett. 115, 161802 (2015).

[59] R. Aaij et al. (LHCb Collaboration), Phys. Rev. D 95, 071101 (2017).

[60] J. P. Lees et al. (BABAR Collaboration), Phys. Rev. Lett. 114, 171801 (2015).

[61] A. Alavi-Harati et al. (KTEV Collaboration), Phys. Rev. Lett. 84, 5279 (2000).

[62] M. J. Dolan, F. Kahlhoefer, C. McCabe, and K. SchmidtHoberg, J. High Energy Phys. 03 (2015) 171; 07 (2015) $103(\mathrm{E})$.

[63] A. V. Artamonov et al. (BNL-E949 Collaboration), Phys. Rev. D 79, 092004 (2009).

[64] M. Acciarri et al. (L3 Collaboration), Phys. Lett. B 385, 454 (1996).

[65] S. Chatrchyan et al. (CMS Collaboration), Phys. Rev. Lett. 109, 121801 (2012).

[66] R. Aaij et al. (LHCb Collaboration), J. High Energy Phys. 08 (2018) 147.

[67] U. Haisch and J. F. Kamenik, Phys. Rev. D 93, 055047 (2016).

[68] R. V. Harlander, S. Liebler, and H. Mantler, Comput. Phys. Commun. 184, 1605 (2013).

[69] A. Berlin, S. Gori, P. Schuster, and N. Toro, Phys. Rev. D 98, 035011 (2018).

[70] M. Anelli et al. (SHiP Collaboration), arXiv:1504.04956.

[71] M. Antinucci, A. Bertin, P. Capiluppi, M. D’AgostinoBruno, A. M. Rossi, G. Vannini, G. Giacomelli, and A. Bussiere, Lett. Nuovo Cimento 6, 121 (1973).

[72] D. E. Groom, Atomic and Nuclear Properties of Materials for more than 300 materials, pdg.lbl.gov/ AtomicNuclearProperties, 2017.

[73] F. Bergsma et al. (CHARM Collaboration), Phys. Lett. B 157B, 458 (1985).

[74] E. Cortina Gil et al. (NA62 Collaboration), J. Instrum. 12, P05025 (2017).

[75] G. Lanfranchi (NA62 Collaboration), Proc. Sci. EPSHEP2017 (2017) 301.

[76] G. Lanfranchi, Sensitivity of the SHiP experiment to a light scalar particle mixing with the Higgs, Report No. CERNSHiP-NOTE-2017-001, 2017, http://cds.cern.ch/record/ 2243034? $1 n=e n$.

[77] A. Fradette and M. Pospelov, Phys. Rev. D 96, 075033 (2017).

[78] J. R. Ellis and K. A. Olive, Phys. Lett. B 193, 525 (1987).

[79] M. S. Turner, Phys. Rev. Lett. 60, 1797 (1988). 
[80] M. Ablikim et al. (BESIII Collaboration), Phys. Rev. D 96, 092007 (2017).

[81] F. Wilczek, Phys. Rev. Lett. 39, 1304 (1977).

[82] J. R. Ellis, K. Enqvist, D. V. Nanopoulos, and S. Ritz, Phys. Lett. 158B, 417 (1985); 163B, 408(E) (1985).

[83] M. I. Vysotsky, Phys. Lett. B 97B, 159 (1980).

[84] P. Nason, Phys. Lett. 175, 223 (1986).

[85] H. E. Haber, G. L. Kane, and T. Sterling, Nucl. Phys. B161, 493 (1979).

[86] J. R. Ellis, M. K. Gaillard, D. V. Nanopoulos, and C. T. Sachrajda, Phys. Lett. 83B, 339 (1979).
[87] B. Batell, M. Pospelov, and A. Ritz, Phys. Rev. D 83, 054005 (2011).

[88] P. Ball and R. Zwicky, Phys. Rev. D 71, 014015 (2005).

[89] P. Ball and R. Zwicky, Phys. Rev. D 71, 014029 (2005).

[90] H. Leutwyler and M. A. Shifman, Nucl. Phys. B343, 369 (1990).

[91] R. S. Willey and H. L. Yu, Phys. Rev. D 26, 3287 (1982).

[92] J. F. Kamenik and C. Smith, J. High Energy Phys. 03 (2012) 090. 